\title{
STATISTICAL CONVERGENCE OF
} ASYMPTOTIC MARTINGALES

\author{
Danjela Braho ${ }^{1}$, Edlira Donefski ${ }^{2}$ \\ ${ }^{1,2}$ Department of Mathematics, Informatics and Physics \\ University F. S. Noli \\ 11, Rilindasit Blvd., Korçë, 7002, ALBANIA
}

\begin{abstract}
Statistical convergence has become an active area of research under the name of statistical convergence since 1990s of the last century. It has appeared in a wide variety of topics such as number theory, measure theory, trigonometric series, summability theory, in the study of strong integral summability and Banach spaces.

In this paper statistical convergence is used to obtain some new results on amarts. Amarts generalize martingales considerably since every convergent sequence of random variables with integrable supremum is an amart. Our goal is the study of statistical convergence of asymptotic martingales of statistical Bochner integrable functions. We obtain some results for the statistical convergence of vector valued uniform amarts without assuming the Radon-Nikodym Property.
\end{abstract}

AMS Subject Classification: 60G48, 40A30, 28B05

Key Words: statistical convergence, amart, statistical Bochner integrable

\section{Introduction}

Amarts generalize martingales; the generalization is considerable since every convergent sequence with integrable supremum is an amart. The amart combines several useful properties of the martingale, submartingale, supermartingale. Thus the class of martingales is closed under linear combinations, the class of supermartingales under infimum, the class of submartingales under supremum; but the class of amarts is closed under all three operations.

$\begin{array}{ll}\text { Received: } & 2017-06-14 \\ \text { Revised: } & 2017-11-15\end{array}$

Published: December 23, 2017

(c) 2017 Academic Publications, Ltd. url: www.acadpubl.eu 
In [1] A. Bellow proved that in contrast to the vector-valued martingale which converges strongly almost everywhere if and only if the Banach space E has the Radon-Nikodym property, the vector-valued asymptotic martingale converges strongly almost everywhere if and only if $\mathrm{E}$ is of finite dimension. During years 70-80's several articles on asymptotic martingales (amarts) have appeared. The first unified treatment was given by Edgar and Sucheston in [8], where further references can be found. Recently Marraffa in [16] has given a characterization of absolutely summing operators by means of McShane integrable stochastic processes. Also Bouzar in [14] had obtained theorems on almost sure convergence for vector-valued uniform amarts.

Statistical convergence recently also has become an active field of research. It seems to have many applications in different fields of mathematics such as in $[9,11,12]$.

In 2012, Caushi and Tato (see [3]) has proposed a new type of integral such as statistical integral of Bochner type. Based in this new type of integral we have studied a new type of martingales such as the martingales of statistical Bochner integrable functions (see $[4,5,6,7]$ ) thus presenting some results on martingales of statistical Bochner and Pettis integrable functions on Banach space.

In Section 3 we give some new results on properties of amarts of statistically Bochner integrable random variables and in Section 4 we present some new results related to uniform amarts of statistically Bochner integrable random variables.

\section{Definitions and Preliminaries}

In this section we recall some basic definitions and notations which form the background of the present work.

Let $\mathrm{E}$ be a Banach space with norm $\|.\| \mathrm{B}(\mathrm{E})$ its unit ball and $\mathrm{E}^{*}$ its dual. A subset $T_{s}$ of $\mathrm{E}^{*}$ is called a total set over $\mathrm{E}$, if $x^{\prime}(x)=0$ for each $x^{\prime} \in T_{s}$ implies $x=0$. Throughout this note $(\Omega, F, \mu)$ is a probability space and $\left(F_{n}\right)_{n \in \mathbb{N}}$ an increasing sequence of sub- $\sigma$-algebras of $F$. Moreover, without loss of generality, we will assume that $F_{\infty}$ is the $\sigma$-algebra generated by $\bigcup_{n \in \mathbb{N}} F_{n}$. We denote by $\mathrm{T}$ the set of the set of bounded stopping times for $\left(F_{n}\right)_{n \in N}$. A stopping time of the sequence $\left(F_{n}\right)_{n \in D}$ (where $D=\mathbb{N}$ or $D=-\mathbb{N}$ ) is a function $\tau: \Omega \rightarrow \mathbb{N} \cup\{+\infty\}$ such that $\{\tau=n\} \in F_{n}$ for all $n \in D$. Let $T=T_{D}$ be the set of all bounded stopping times. For each $\tau \in T$, we associate the $\sigma$-algebra $F_{\tau}=\left\{A \in F_{\infty}: A \cap\{\tau=n\} \in F_{n}, \forall n \in \mathbb{N}\right\}$. 
A random variable is any mapping $\mathrm{X}$ from $\Omega$ into $\mathrm{E}$. Unless specified otherwise, all random variables in the sequel are E-valued.

Definition 1. The sequence $\left(X_{n}\right)_{n \in D}$ is said to be an amart for $\left(F_{n}\right)_{n \in D}$ iff $s t-\int\left|X_{n}\right|<\infty$ for all $n \in D$ and $\left(s t-\int X_{\tau}\right)_{\tau \in T}$ converges.

Definition 2. An adapted sequence $\left(X_{n}, F_{n}, n \in \mathbb{N}\right)$ of random variables is said to be

a) Weak amart if the net $\left(E\left(X_{\tau}\right), \tau \in T\right)$ converges statistically weakly in E.

b) A uniform weak amart if it is a weak amart such that for any $A \in$ $\bigcup_{n \in \mathbb{N}} F_{n}, E\left(X_{n} I_{A}\right)$ converges statistically weakly in E.

We follow the notions about the statistical convergence of sequences introduced by Fridy $[12,13]$ as well as the approach of Schoenberg [10] about integration.

A subset $\mathrm{A}$ of the ordered set $\mathbb{N}$ of natural numbers is said to have density $\delta(A)$, if $\lim \frac{|A(n)|}{n}=\delta(A)$, where $A(n)=\{k<n: k \in A\}$ and $|A|$ denotes the cardinality of set $A \subset \mathbb{N}$. It is clearly that finite sets have the density zero. If a property $P(k)=\{k ; k \in A\}$ holds for all $k \in A$ with $\delta(A)=1$, we say that property $\mathrm{P}$ holds for all $\mathrm{k}$ that is a.a.k (allmost all natural $\mathrm{k}$ ).

Definition 3. The vectorial sequence $x_{n}$ is statistically convergent (stconvergent) to the vector $\mathrm{L}$ of a vectorial normed space if for each $\varepsilon>0$

$$
\lim _{n \rightarrow \infty} \frac{1}{n}\left|\left\{k \leq n:|| \mathrm{x}_{k}-L \| \geq \varepsilon\right\}\right|=0,
$$

i.e. $\left\|\mathrm{x}_{k}-L\right\|<\varepsilon$, a.a.k.

Lemma 4. (Lemma Salat [15] ) A sequence $\left(x_{k}\right)$ is statistically convergent to $L$ if and only if there exists a set

$$
K=\left\{k_{1}<k_{2}<\ldots<\right\} \subset \mathbb{N}
$$

that $\delta(K)=1$ and

$$
\lim _{n \rightarrow \infty}\left(x_{k_{n}}\right)=L
$$

The set $\mathrm{K}$ (mentioned in the lemma 4) is directed and the sequence $\left(x_{k_{n}}\right)$ is called the essential subsequence of $\left(x_{k}\right)$. The above lemma can be reworded: A sequence $\left(x_{k}\right)$ is statistically convergent to $\mathrm{L}$ if and only if there exists an essential subsequence $\left(x_{k_{n}}\right)$ which converges in usual meaning to the limit L.

We can now formulate a new lemma of Salat's lemma [15] for a sequence of functions with value in vectorial space: The sequence $\left\{f_{k}(x)\right\}$ where $f_{k}: S \rightarrow X$ 
(X a vectorial normed space) is statistically convergent to $f(x)$, if and only if, there exists an essential subsequence $f_{k_{n}}$ of it that is convergent to $f(x)$.

Let $\left\{f_{k}\right\}$ be a sequence of functions with value in vectorial space. For each $x$ of the domain, we consider the functional sequence $\left(f_{k}(x)\right)$. Let $(X,\|\cdot\|)$ be a Banach space and $(\Omega, \Sigma, \mu)$ is a finite measure space.

Definition 5. [3] A function $f: \Omega \rightarrow X$ is called statistical Bochner integrable if there exists a st- Cauchy sequence of simple functions $\left(f_{k}\right)$ such that:

a) statistically convergent almost everywhere by $\mu$

b) $s t-\lim _{k} \int_{\Omega}\left\|f_{k}-f_{N}\right\| d \mu=0$ almost everywhere

and

$$
s t-\lim _{n} \int_{\Omega} f_{n} d \mu
$$

is called st- Bochner integral.

The set of statistical Bochner integrable functions is a linear space.

\section{Some Properties for Amarts}

In this section we would obtain some results for amarts of statistical Bochner inegrable random variables. Let start with a lemma for amarts. In which we extend to amarts of statistically Bochner integrable random variables a characterization known in the case of Bochner integrable random variables.

Lemma 6. Let $\left(X_{n}\right)_{n \in D}$ be an amart for $\left(F_{n}\right)_{n \in D}$. Then $\left(s t-\int X_{\tau}\right)_{\tau \in T}$ is statistically bounded.

Proof. We prove the case $D=\mathbb{N}$; the other case is the same. Since $\left(s t-\int X_{\tau}\right)_{\tau \in T}$ converges statistically, there is $N \in \mathbb{N}$ such that

$$
\left|s t-\int X_{N}-\left(s t-\int X_{\tau}\right)\right| \leq 1
$$

for all $\tau \geq N$.

If $\tau$ is any bounded stopping time, then

$$
\left|s t-\int X_{\tau \wedge N}\right| \leq \int \max _{1 \leq n \leq N}\left|X_{n}\right|
$$

and

$$
\left|s t-\int X_{\tau \wedge N}-\left(s t-\int X_{N}\right)\right| \leq 1
$$


so

$$
\begin{aligned}
& \left|s t-\int X_{\tau}\right|=\left|\left(s t-\int X_{\tau \wedge N}\right)+\left(s t-\int X_{\tau \wedge N}\right)-\left(s t-\int X_{N}\right)\right| \\
& \leq \int \max _{1 \leq n \leq N}\left|X_{n}\right|+1
\end{aligned}
$$

Therefore $\sup _{T}\left|s t-\int X_{\tau}\right|<\infty$.

It is clear that a linear combination of amarts is an amart. Also it is clear that the maximum and minimum are also amarts.

The proposition below may be considered as an extension of the case of amarts of Bochner integrable random variables to amarts of statistically Bochner integrable random variables. The proof of which follows with suitable changes.

Proposition 7. Let $\left(X_{n}\right)_{n \in D}$ and $\left(Y_{n}\right)_{n \in D}$ be sequences adapted to $\left(F_{n}\right)_{n \in D}$. If assume in addition that they are $L_{1}$-bounded. Then:

(a) If $\left(s t-\int X_{\tau}\right)_{\tau \in T}$ and $\left(s t-\int Y_{\tau}\right)_{\tau \in T}$ are statistically bounded above (below) then $\left(s t-\int\left(X_{\tau} \vee Y_{\tau}\right)\right)_{\tau \in T}$ and $\left(s t-\int\left(X_{\tau} \wedge Y_{\tau}\right)\right)_{\tau \in T}$ are statistically bounded above (below).

(b) If $\left(X_{n}\right)_{n \in D}$ and $\left(Y_{n}\right)_{n \in D}$ are amarts, then $\left(X_{n} \vee Y_{n}\right)_{n \in D}$ and $\left(X_{n} \wedge Y_{n}\right)_{n \in D}$ are amarts.

Lemma 8. Let $\left(X_{n}\right)_{n \in \mathbb{N}}$ be an amart, $\sigma$ a stopping time. Then $\hat{X}_{n}=X_{n \wedge \sigma}$ is an amart.

Proof. Let apply Proposition 7 with $\tau_{n}=n \wedge \sigma$. In our case is easy to see that $F_{\tau_{n}} \subseteq F_{n}, n \in \mathbb{N}$ and $\left(\hat{X}_{n}, F_{n}\right)$ is an amart.

\section{Main Result}

We start out with the case of uniform weak amarts. The following proposition extends to amarts of statistically Bochner integrable random variables a result known for Bochner integrable random variables.

Proposition 9. Let $\left(X_{n}, n \in \mathbb{N}\right)$ be a uniform weak amart of statistically Bochner integrable random variables. Assume that there exist a random variable $X$ and a total subset $T_{s}$ of $E^{*}$ such that for each $x^{\prime} \in T_{s},\left(x^{\prime} \circ X_{n}, n \in \mathbb{N}\right)$ convergence a.s. to $x^{\prime} \circ X$. Then $\left(X_{n}, n \in \mathbb{N}\right)$ is statistically convergent to $X$.

Proof. Since $\left(X_{n}, n \in \mathbb{N}\right)$ and $\mathrm{X}$ are a.s separably valued, we may and do assume that $\mathrm{E}$ is separable. It can be easily seen that $\left(X_{\sigma \wedge n}, F_{\sigma \wedge n}, n \in \mathbb{N}\right)$ is a uniform weak amart. Therefore, by the maximal inequality of Chacon and 
Sucheston (1975) and a classical stopping time argument, we can assume that $E\left(\sup _{n}\left\|X_{n}\right\|\right)<\infty$. This implies that the (finitely additive) set function

$$
\mu(A)=s t-\lim _{n \rightarrow \infty} E\left(X_{n} I_{A}\right), \mathrm{A} \in \bigcup_{n \in \mathbb{N}} F_{n}
$$

is absolutely continuous and is of bounded variation. Its extension to $F_{\infty}$, which we also denote by $\mu$, satisfies

$$
\mu(A)=s t-\lim _{n \rightarrow \infty} E\left(X_{n} I_{A}\right), \mathrm{A} \in F_{\infty}
$$

Letting $A_{k}=[\|X\| \leq k], k \in \mathbb{N}$, and noting that $X I_{A_{k}}$ is statistical Bochner integrable, it follows that for any $x^{\prime} \in T_{s}$

$$
\begin{aligned}
& x^{\prime} \circ \mu\left(A_{k}\right)=s t-\lim _{n \rightarrow \infty} E\left(\left(x^{\prime} \circ X_{n}\right) I_{A_{k}}\right) \\
& =E\left(\left(x^{\prime} \circ X\right) I_{A_{k}}\right)=x^{\prime}\left(E\left(X I_{A_{k}}\right)\right)
\end{aligned}
$$

Since $T_{s}$ is total, we have

$$
\mu\left(A_{k}\right)=s t-\lim _{k} E\left(X I_{A_{k}}\right), k \in \mathbb{N}
$$

which implies that

$$
s t-\lim _{k} E\left(\|X\| I_{A_{k}}\right)=\|\mu\|\left(A_{k}\right) \leq E\left(\sup _{n}\left\|X_{n}\right\|\right)
$$

which in turn implies that $\mathrm{X}$ is st-Bochner integrable. Repeating the same argument as above, we also have

$$
\mu(A)=E\left(X I_{A}\right), A \in F_{\infty}
$$

Now for each $x^{\prime} \in E^{*},\left(x^{\prime} \circ X_{n}, n \in \mathbb{N}\right)$ is an $L^{1}$-dominated, real-valued amart, therefore $x^{\prime} \circ X_{n}$ converges a.s. and for $A \in F_{\infty}$

$$
\begin{aligned}
& E\left(\left(x^{\prime} \circ X\right) I_{A}\right)=x^{\prime}\left(E\left(X I_{A}\right)\right) \\
& =s t-\lim _{n \rightarrow \infty} E\left(\left(x^{\prime} \circ X_{n}\right) I_{A}\right) \\
& =E\left(s t-\lim _{n \rightarrow \infty}\left(x^{\prime} \circ X_{n}\right) I_{A}\right)
\end{aligned}
$$

which implies that $x^{\prime} \circ X=s t-\lim _{n \rightarrow \infty} x^{\prime} \circ X_{n}$.

Since weak sequential amarts and strong amarts are themselves uniform weak amarts, we have two corollaries which proof follows with suitable changes. 
Corollary 10. Let $\left(X_{n}, n \in \mathbb{N}\right)$ be a weak sequetial amart of statistically Bochner integrable random variables. Assume that there exist a random variable $X$ and a total subset $T_{s}$ of $E^{*}$ such that for each $x^{\prime} \in T_{s},\left(x^{\prime} \circ X_{n}, n \in \mathbb{N}\right)$ convergence a.s. to $x^{\prime} \circ X$. Then $\left(X_{n}, n \in \mathbb{N}\right)$ converges statistically to $X$.

Corollary 11. Let $\left(X_{n}, n \in \mathbb{N}\right)$ be a strong amart of statistically Bochner integrable random variables. Assume that there exist a random variable $X$ and a total subset $T_{s}$ of $E^{*}$ such that for each $x^{\prime} \in T_{s},\left(x^{\prime} \circ X_{n}, n \in \mathbb{N}\right)$ convergence a.s. to $x^{\prime} \circ X$. Then $\left(X_{n}, n \in \mathbb{N}\right)$ converges statistically to $X$.

\section{References}

[1] A. Bellow, On vector-valued asymptotic martingales, Proc. Natl. Acad. Sci. USA, 73, No. 6 (1976), 1798-1799

[2] A. Bellow, Stability properties of the class of asymptotic martingales, Bulletin of the American Mathematical Society, 82, No. 2 (1976), 338-340

[3] A. Caushi, A. Tato, A statistical integral of Bochner type on Banach space, Hikari Ltd Appl. Math. Sci., 6, No. 137-140 (2012), 6857-6870.

[4] D. Braho, A. Caushi, On the Statistical Convergence of Martingales, Hikari Ltd Appl. Math. Sci., 9, No. 17(2015), 821- 830.

[5] D. Braho, E. Donefski, On the martingale statistical convergence, Aktet, Journal of Institute Alb-Shkenca, 9, No. 1 (2016), 46-49

[6] D. Braho, A. Tato, Martingales via statistical convergence, Journal of Advances in Mathematics, 12, No. 7 (2016), 6402-6406.

[7] D. Braho, A. Tato, Statistical Convergence of Banach Valued Martingales, ISJ, Journal of Mathematics, 2 (2016), 71-75

[8] G. A. Edgar and L. Sucheston, Amarts: A class of asymptotic martingales, A. Discrete parameter, J. Multivariate Anal, 6 (1976), 193-221.

[9] H. Çakalli, A study on statistical convergence, Functional analysis, approximation and computation 1:2 (2009), 19-24.

[10] I. J. Schoenberg, The integrability of certain functions and related summability methods, The American Mathematical Monthly, 66, No. 5 (1959), 361-375.

[11] J. A. Fridy, On statistical convergence. Analysis, 5, No. 4 (1985), 301-313.

[12] J. A. Fridy, Statistical limit points. Proceedings of the American Mathematical Society, 118, No. 4 (1993), 1187-1192.

[13] J.A. Fridy, C. Orhan, Statistical limit superior and limit inferior, Proc. Amer. Math. Soc., 125, No. 12 (1997), 3625-3631.

[14] N. Bouzar, On almost sure convergence without the Radon-Nikodym property, Acta Math. Univ. Comenianae, LXX, No. 2 (2001), 167-175

[15] T. Salat, On statistically convergent sequences of real numbers, Math. Slovaca, 30 (1980), 139-150. 
[16] V. Marraffa, On weakly measurable stochastic processes and absolutely summing operators, 131 Mathematica Bohemica, No. 4 (2006), 379-391 